BULGARIAN ACADEMY OF SCIENCES

CYBERNETICS AND INFORMATION TECHNOLOGIES • Volume 14, Special Issue

Sofia $\bullet 2014$

Print ISSN: 1311-9702; Online ISSN: 1314-4081

DOI: $10.2478 /$ cait-2014-0046

\title{
Study of Algorithms of Oil Immersed Transformer Temperature Measurement Technology
}

\section{Danping Jia, Ximeng Gao, Chunhua Li}

School of Information Science and Engineering, Shenyang University of Technology, No 111 Shenliao West Road Economic\&Technological Development Zone Shenyang 110870, China

Email:winnerjia@sohu.com

\begin{abstract}
In the paper presented the temperature of an oil-immersed transformer was measured, based on the principles of the fluorescence afterglow life. Three methods were used to calculate the fluorescence afterglow life $\tau$ by using the least squares method, the integral area ratio method and Prony algorithm. The leastsquare method, the integral area ratio method and the program of Prony algorithm are written using Matlab and $C++$. The Least-square fitting is susceptible to the influence of the DC component. When the DC location is different, the fluorescence afterglow life $\tau$ values vary widely. The integral area ratio method is not influenced by DC component, but it has low sensitivity. Prony algorithm is not affected by DC, it has high sensitivity. So Prony algorithm is selected as a way to obtain the fluorescence afterglow lifetime value $\tau$.
\end{abstract}

Keywords: Temperature, the least squares, integral area ratio, Prony algorithm, fluorescence afterglow life.

\section{Introduction}

With the rapid development of our society, there appears a higher demand for the corresponding device security $[1,2]$. The reliability of the power system needs to be assured. The transformer is the main equipment in the power transmission and transformation system, which is a kind of electrical equipment to realize $\mathrm{AC}$ power conversion. Insulation ability is one of the important factors to the life of the 
transformer. The main factor that affects the ability of the transformer insulation is the temperature of the transformer winding. So the monitoring technology of transformer winding is the focus of the related research on the transformer. In the present transformer the oil temperature and the temperature of the transformer winding are the most widely used measurements, but due to measuring the values of average temperature, to reflect the transformer operation has certain limitations in accuracy in real time $[3,4]$. Now there are two detection methods of oil-immersed transformer winding temperature. They are the direct measurement and the indirect measurement method. The indirect measurement method is an estimating simulation method. The simulation results are a little different from the real situation. The direct measuring method can put the sensors in the interior of the transformer, do real-time measurements and the measurement results are accurate. So the direct measurement method which is used to monitor the performance of the transformer is the important trend in future. Precise temperature measurement is very important.

\section{Introduction of the fluorescence afterglow signal}

Since the fluorescence lifetime and temperature is the essence, that has nothing to do with light intensity, so the formulation of the fluorescence decay curve is

$$
I(t)=I_{0} e^{-\frac{t}{\tau}}+I_{d}+I_{n},
$$

where $\tau$ is the intensity of the fluorescence afterglow, $I_{n}$ is the random noise, $I_{d}$ is the DC component of the system [5].

The fluorescence afterglow signal is collected continuously by the data acquisition card. According to previous experience, the collected signal voltage must be the close index curve. The decay time is calculated through the index curve. The attenuation time of the signal has a certain corresponding relationship with temperature. Thus the measured temperature is obtained by using the fluorescence afterglow lifetime [6-8]. There are three kinds of common methods to calculate the fluorescence afterglow lifetime, namely the least squares fitting, integral area ratio method, Prony algorithm.

\section{The least squares fitting method}

The method of the Least-squares fitting is a common method which is used to calculate the fluorescence lifetime. Its eliminating the DC component mathematical model is an exponential function aimed to find the exponential curve which has the least squares error with the measurement data of the measured afterglow through the method of Least-squares fitting. The exponential curve is used to calculate the fluorescence lifetime. The method focuses on the overall law of the signal and has a good effect on suppressing the partial disturbances of the measured signal and random noise. 
For ease of fitting the processed signal which is a single exponential is given in (2)

$$
I(t)=I_{0} \exp \left(-\frac{t}{\tau}\right)
$$

Making variable substitutions into the linear equation, like the natural logarithm which is used in (2), it is obtained:

$$
\ln I(t)=\ln \left[I_{0} \exp \left(-\frac{t}{\tau}\right)\right]=\ln I_{0}-\frac{t}{\tau}
$$

If $\ln I_{0}=a,-\frac{1}{\tau}=b, \ln I(t)=y(t)$, (3) can be written as

$$
y(t)=a+b t .
$$

This is a linear equation. It can calculate the data requirements of the afterglow of the closest line by using the method of the least-squares linear fitting. The algorithm is as follows:

By measuring the data $\left(t_{i}, I_{\left(t_{i}\right)}\right), \quad i=1,2, \ldots, n$, calculate $y_{\left(t_{i}\right)}=\ln \left(I_{\left(t_{i}\right)}\right)$, and then according to the definition of the least-squares method, the following is obtained:

$$
\begin{gathered}
S_{0}=\sum_{i=1}^{n} t_{i}^{0}, S_{1}=\sum_{i=1}^{n} t_{i}^{1}, S_{2}=\sum_{i=1}^{n} t_{i}^{2}, \\
T_{0}=\sum_{i=1}^{n} y\left(t_{i}\right) t_{i}^{0}, T_{1}=\sum_{i=1}^{n} y\left(t_{i}\right) t_{i}^{1} .
\end{gathered}
$$

The normal equations are:

$$
\left\{\begin{array}{l}
S_{0} a+S_{1} b=T_{0}, \\
S_{1} a+S_{2} b=T_{1} .
\end{array}\right.
$$

Solving (6), $a, b$ is given by

$$
a=\frac{T_{1} S_{1}-T_{0} S_{2}}{S_{1}^{2}-S_{2} S_{0}} ; b=\frac{T_{0} S_{1}-T_{1} S_{0}}{S_{1}^{2}-S_{2} S_{0}} .
$$

If (7) substitutes (6) a fitting straight line equation is given by

$$
y(t)=\frac{T_{1} S_{1}-T_{0} S_{2}}{S_{1}^{2}-S_{2} S_{0}}+\frac{T_{0} S_{1}-T_{1} S_{0}}{S_{1}^{2}-S_{2} S_{0}} t .
$$

Taking the index $e$ on both sides in (8), the form of the fitting exponential curve can be resumed:

$$
\exp [y(t)]=I(t)=\exp \left(\frac{T_{1} S_{1}-T_{0} S_{2}}{S_{1}^{2}-S_{2} S_{0}}\right) \cdot \exp \left(\frac{T_{0} S_{1}-T_{1} S_{0}}{S_{1}^{2}-S_{2} S_{0}} t\right) .
$$

Now the following equation can be obtained:

$$
y=-A x+B .
$$

The advantage of the least squares fitting is a better fitting effect. By using the method, the unknown data is calculated. The sum of the squares errors is the smallest between the actual data and the calculated data. So the fitting effect of the best approximation can be achieved. 


\section{Integral area ratio methods}

The integration method is the method which is earlier proposed. Equation (1) is the processing model, it can improve the signal strength and smooth noise, which is sensitive to the DC. In the paper, the integral ratio method is proposed as a mathematical model of the practical application of (1). It maintains the original advantages and avoids the influence of the DC component. Its processing time is shorter than the fluorescence time [10].

The principle of the integral area ratio method: A part of the exponential curve is divided into three parts at an equal time interval. Each part is integral respectively, as shown in Fig. 1.

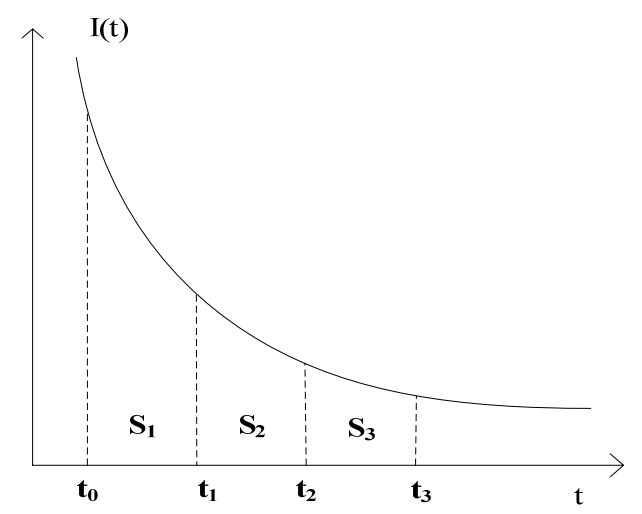

Fig. 1. Principle of integral area ratio method

The results of the fluorescence curves are integrated three times:

$$
\begin{aligned}
& S_{1}=\int_{t_{1}}^{t_{2}}\left[I_{0} \exp \left(-\frac{t}{\tau}\right)+I_{d}+I_{n}\right] d t=-I_{0} \tau\left[\exp \left(-\frac{t_{2}}{\tau}\right)-\exp \left(-\frac{t_{1}}{\tau}\right)\right]+I_{d}\left(t_{2}-t_{1}\right), \\
& S_{2}=\int_{t_{2}}^{t_{3}}\left[I_{0} \exp \left(-\frac{t}{\tau}\right)+I_{d}+I_{n}\right] d t=-I_{0} \tau\left[\exp \left(-\frac{t_{3}}{\tau}\right)-\exp \left(-\frac{t_{2}}{\tau}\right)\right]+I_{d}\left(t_{3}-t_{2}\right), \\
& S_{3}=\int_{t_{3}}^{t_{4}}\left[I_{0} \exp \left(-\frac{t}{\tau}\right)+I_{d}+I_{n}\right] d t=-I_{0} \tau\left[\exp \left(-\frac{t_{4}}{\tau}\right)-\exp \left(-\frac{t_{3}}{\tau}\right)\right]+I_{d}\left(t_{4}-t_{3}\right) .
\end{aligned}
$$

The average effect of the integral makes random noise which is reduced greatly by the type, so it is rounded and does not count.

Each time the interval is

$$
t_{3}-t_{2}=t_{2}-t_{1}=t_{1}-t_{0}=\Delta T,
$$

$\Delta \mathrm{T}$ is the integral width of each integration. The following ratio can be obtained:

$$
\frac{S_{1}-S_{2}}{S_{2}-S_{3}}=\frac{e^{-\frac{t_{0}}{\tau}}\left[1+e^{-\frac{2 \Delta T}{\tau}}-2 e^{-\frac{\Delta T}{\tau}}\right]}{e^{\frac{t_{1}}{\tau}}\left[1+e^{-\frac{2 \Delta T}{\tau}}-2 e^{-\frac{\Delta T}{\tau}}\right]}=e^{\frac{\Delta T}{\tau}} .
$$


For $\frac{S_{1}-S_{2}}{S_{2}-S_{3}}=B$, so that (13) is also written as

$$
B=e^{\frac{\Delta T}{\tau}} ; \tau=\Delta T / \ln B
$$

The integral ratio method has two advantages:

1) The method is simple. It is easy to implement it on a computer. It requires less computer memory and less time.

2) It is not needed to calculate DC component Id singly, it only takes about 2 to 3 times of the measurement time of the fluorescence lifetime. It can shorten the processing cycle and save the measurement time greatly. It also quickens the speed of measurement and real-time. There are more advantages in the system which require a fast speed of response.

\section{Prony algorithm}

Prony algorithm is used to fit the sample data by using a linear combination of the exponential function [11-14]. The extended Prony algorithm uses the mathematical model with an exponential function of $p$ arbitrary parameters - amplitude, phase, frequency and attenuation factor. The function form of the discrete time is

$$
\hat{x}(n)=\sum_{i=1}^{p} b_{i} z_{i}^{n}, n=0,1, \cdots, N-1
$$

$x(n)$ is used as an approximation of $x(n)$, assuming that $b_{i}$ and $z_{i}$ are plural in (15).

$$
\begin{aligned}
& b_{i}=A_{i} e, \\
& z_{i}=A_{i} e^{\left[\left(\alpha_{i}+j 2 \pi f_{i}\right) \Delta t\right] j \theta_{i}},
\end{aligned}
$$

where $A_{i}$ is the amplitude, $\theta_{i}$ is the phase (its unit is radians), $\alpha_{i}$ is the attenuation factor (fluorescence lifetime), $f_{i}$ is the oscillation frequency, $\Delta t$ is the sampling interval,

$$
\varepsilon=\sum_{n=0}^{N-1}|x(n)-\hat{x}(n)|^{2} .
$$

The derived differential equation is

$$
x(n)=-\sum_{i=1}^{p} \alpha_{i} x(n-i), \quad n=0,1, \cdots, N-1 .
$$

In order to establish Prony fitting method, the linear matrix equation has been estimated using the least squares method. It is shown in 


$$
\begin{gathered}
\left(\begin{array}{ccc}
x(p) & \cdots & x(0) \\
\vdots & \ddots & \vdots \\
x(N-1) & \cdots & x(N-p-1)
\end{array}\right)\left(\begin{array}{l}
1 \\
\vdots \\
\alpha_{p}
\end{array}\right)=\left(\begin{array}{l}
\varepsilon(0) \\
\vdots \\
\varepsilon(N-1)
\end{array}\right), \\
\gamma(i, j)=\sum_{n=p}^{N-1} x(n-j) x^{*}(n-i), \quad i, j=0,1, \cdots, P .
\end{gathered}
$$

The fitting equation of Prony method can be obtained

$$
\left(\begin{array}{ccc}
\gamma(0,0) & \cdots & \gamma(0, p) \\
\vdots & \ddots & \vdots \\
\gamma(p, 0) & \cdots & \gamma(p, p)
\end{array}\right)\left(\begin{array}{l}
1 \\
\vdots \\
\alpha_{p}
\end{array}\right)=\left(\begin{array}{l}
\varepsilon(p) \\
\vdots \\
0
\end{array}\right) .
$$

The coefficients $\alpha_{1}, \ldots, \alpha_{p}$ estimation can be obtained by calculating the equation. When $\alpha_{1}, \alpha_{p}$ are obtained, the characteristic polynomial can also be obtained:

$$
1+\alpha_{1} z^{-1}+\ldots+\alpha_{p} z^{-p}=0 .
$$

$Z_{i}$ are Prony poles. Then the index model of (15) is simplified to a linear equation of the unknown parameters $b_{i}$ :

$$
\left(\begin{array}{ccc}
1 & \cdots & 1 \\
\vdots & \ddots & \vdots \\
Z_{1}^{n-1} & \cdots & Z_{P}^{n-1}
\end{array}\right)\left(\begin{array}{l}
b_{1} \\
\vdots \\
b_{p}
\end{array}\right)=\left(\begin{array}{l}
\hat{x}(1) \\
\vdots \\
\hat{x}(p)
\end{array}\right) .
$$

The least-square solutions can be obtained by solving this equation:

$$
b=\left(Z^{H} Z\right)^{-1} Z^{H} \hat{x} \text {. }
$$

Its four parameter values are:

$$
\begin{gathered}
A_{i}=\left|b_{i}\right|, \\
\theta_{i}=\arctan \left[\operatorname{Im}\left(b_{i}\right) / \operatorname{Re}\left(b_{i}\right)\right] /(2 \pi \Delta t), \\
\alpha_{i}=\ln \left|Z_{i}\right| / \Delta t, \\
f_{i}=\arctan \left[\operatorname{Im}\left(Z_{i}\right) / \operatorname{Re}\left(Z_{i}\right)\right] /(2 \pi \Delta t) .
\end{gathered}
$$

Prony algorithm has the following advantages:

1) Prony algorithm has its own advantages. The value of the four parameters amplitude, phase, damping ratio (the fluorescence lifetime) and frequency can be directly calculated from the time domain signal, which makes the amount of calculation largely reduced.

2) Prony algorithm does not need to solve the complex characteristic equation and estimate the sample autocorrelation. It only needs to solve the linear equation and several polynomial equations. The signal information can be concluded. It can reduce the amount of calculation and improve the system accuracy. It is a nonlinear multidimensional filter method. 


\section{Selection of the algorithm}

The mathematical model of the Least-squares fitting, eliminating the DC component is an exponential function that finds the exponential curve which has the least squares error with the measurement data of the measured afterglow by representing the afterglow measurement data through the method of Least-squares fitting [15]. The exponential curve is used to calculate the fluorescence lifetime. The method focuses on the overall law of the signal and has a good effect on suppressing the partial disturbances of the measured signal and random noise [16]. Calculating the DC principle of the Least-squares fitting method is shown in Fig. 2.

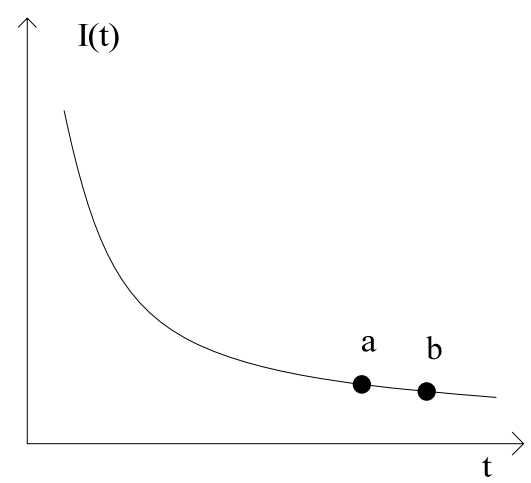

Fig. 2. Calculating DC principle of the Least-squares fitting method

In the figure the curve is the exponential decay curve of the fluorescence afterglow. In the interval of excitation the light pulse of the fluorescence cannot decay to zero. So the approximation of the direct current is calculated by the last paragraph of the fluorescent afterglow data values obtained. In the figure the fluorescence data between $a$ and $b$ were used to get the average $I$. Therefore, $I$ is regarded as direct current and must be removed before fitting.

In Table 1 the least squares method is largely influenced by the impact of the DC component. When DC is taken in different positions, the error of results can reach $13.52 \%$. The error is very large. That is why the Least-squares fitting method cannot be used to calculate the fluorescence lifetime $\tau$.

Table 1. Fluorescence lifetime $\tau$ of different DC positions

\begin{tabular}{|c|c|}
\hline Position of DC (count) & $\tau$ after being removed DC $(\mu \mathrm{s})$ \\
\hline $390-410$ & 569.2 \\
\hline $800-820$ & 550.2 \\
\hline $195-205$ & 501.4 \\
\hline
\end{tabular}

The integral area ratio method is used to calculate the Fluorescent afterglow lifetime $\mu$ s value. The measured temperature is $20-45^{\circ} \mathrm{C}$. The $\tau$ value is shown in Table 2. 
Table 2. Fluorescence lifetime at different temperatures

\begin{tabular}{|c|c|}
\hline Measured temperature $\left({ }^{\circ} \mathrm{C}\right)$ & The fluorescence lifetime $\tau(\mu \mathrm{s})$ \\
\hline 20 & 862.61 \\
\hline 25 & 860.93 \\
\hline 30 & 858.15 \\
\hline 35 & 856.13 \\
\hline 40 & 852.81 \\
\hline 45 & 850.46 \\
\hline
\end{tabular}

We can see that the change of the fluorescence lifetime $\tau$ is $11.95 \mu \mathrm{s}$, when the measured temperature varied from $20{ }^{\circ} \mathrm{C}$ to $45{ }^{\circ} \mathrm{C}$, when the temperature varied by $1{ }^{\circ} \mathrm{C}$; the fluorescence lifetime $\tau$ varied to $0.48 \mu \mathrm{s}$. The change is too small, so that the accuracy of the system becomes low.

For the same data Prony algorithm is used to calculate the fluorescence lifetime $\tau$. The measured temperature is $20-45^{\circ} \mathrm{C}$. The $\tau$ value is shown in Table 3 .

Table 3. Fluorescence lifetime at different temperatures

\begin{tabular}{|c|c|}
\hline Measured temperature $\left({ }^{\circ} \mathrm{C}\right)$ & The fluorescence lifetime $\tau(\mu \mathrm{s})$ \\
\hline 20 & 683.84 \\
\hline 25 & 678.00 \\
\hline 30 & 663.26 \\
\hline 35 & 643.21 \\
\hline 40 & 630.48 \\
\hline 45 & 625.59 \\
\hline
\end{tabular}

The relationship between the temperature and the fluorescent lifetime is shown in Fig. 3.

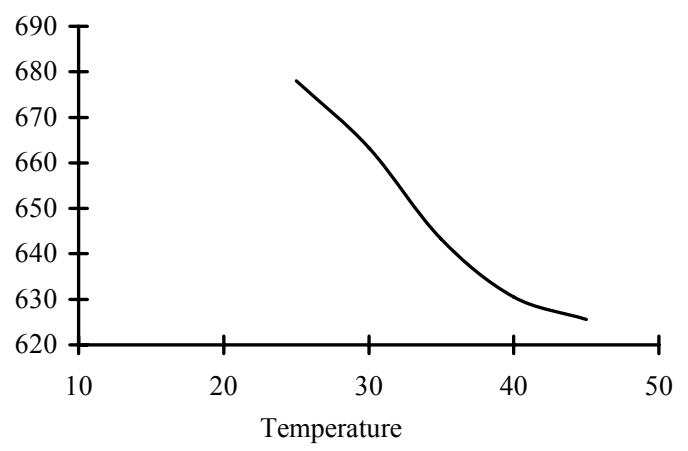

Fig. 3. The relationship between the temperature $\left({ }^{\circ} \mathrm{C}\right)$ and the fluorescence lifetime $(\mu \mathrm{s})$

We can see that the change of the fluorescence lifetime $\tau$ is $58.25 \mu \mathrm{s}$, when the measured temperature varied from 20 to $45^{\circ} \mathrm{C}$. When the temperature varied by $1{ }^{\circ} \mathrm{C}$, the fluorescence lifetime $\tau$ varied to $2.33 \mu$ s. Thus the accuracy of the system is improved about 4 times. The change is very large compared to the integral area ratio method, that is why the accuracy of the system will improve largely. In the paper the processing method of calculating the fluorescent lifetime is used as Prony algorithm. 


\section{Conclusion}

Three data processing algorithms were introduced in the paper, namely the least squares method, the integral area ratio method and Prony algorithm. The basic principle is introduced. Least-square fitting is susceptible to the influence of the DC component. The experiments prove that DC location is different, the maximum of the measured temperature error is $13.52 \%$. Though the integral area ratio method is not affected by DC component, the fluorescent afterglow lifetime $\tau$ value changed to $11.95 \mu \mathrm{s}$, when the temperature changed to $25^{\circ} \mathrm{C}$. Prony algorithm is used to calculate the fluorescence lifetime $\tau$. We can see that the change of the fluorescence lifetime $\tau$ is $58.25 \mu \mathrm{s}$. The accuracy of the system is improved approximately 4 times. That is why we use Prony algorithm to calculate the fluorescence lifetime $\tau$.

Acknowledgments: This work was supported by: National Natural Science Foundation of China under Grant No 61100091; Technology Research Project of Liaoning Province Education Administration under Grant No L2013059; Research Fund for a Doctoral Program of Shenyang University of Technology. The authors would like to express the greatest appreciation and thanks to the reviewers for their detailed reviews and constructive comments, which helped in the improvement of this paper.

\section{References}

1. Danping, Jia, Lin Qiu, Song Lin, Jihua Yang. Research on Temperature Monitoring System for Electrical Equipment Based on Wireless Sensor Networks. - Applied Mechanics and Materials, Vol. 128-129, 2012, 706-709.

2. Danping, Jia, Hongli San, We iq i Yuan, Yingwen Lin. Current Transformer Based On Optical Fiber Thermometry. - Transcations of China Electrotechnical Society, Vol. 23, 2008, No 10, 47-51.

3. Hongjun, Xia. Large Oil-Immersed Transformer Temperature Monitoring Technology. Operation \& Maintenance, Vol. 263, 2009, No 4, 23-24.

4. Shaojie, Wang, Zexi Hua, Li Wang. Design of Online Monitoring System for Thermal Characteristics of Oil Immersed Transformer. - Computer and Modernization, Vol. 192, 2011, No 8, 110-113.

5. Guangli, Zhou, Shulin E, Wenyuan Deng. Development and Application of Optical Fiber Temperature Sensor. - Optical Communication Technology, 2007, No 6, 54-57.

6. Danping, Jia, Ting Jia, Weiqi Yuan, Yingwen Lin. Non-Exponential Component of Fluorescence Decay and its Processing. - Chinese Journal of Scientific Instrument, Vol. 29, 2008, No 12, 2581-2586.

7. Danping, Jia, Lele Lin, Weiqi Yuan, Yingwen Lin. A Study for Stability of High Precision Optical Fiber Fluorescent Temperature Measurement System. - Acta Metrologica Sinica, Vol. 29, 2008, No 2, 129-133.

8. Danping, Jia, Ting Jia, Lu Gao, Y ingwen Lin. Optimal Design of Optical Fiber Fluorescent Thermometry. - In: Proc. of SPIE, 7156, 2008. Doi 1117/12.896.808.

9. Danping, Jia, Peng Gao, Hongli San. Data Processing for Temperature Measurement Based on Fluorescence Lifetime. - Journal of Shenyang University of Technology, Vol. 28, 2006, No 5, 542-545.

10. Danping, Jia, Zhuo Yuan, We i Lin. Research of DC Current Transformer Based on Optical Fiber Thermometry. - J. Nanoelectronics and Optoelectronics, Vol. 7, 2012, No 2, 128-131. 
11. Q i ang Wang, Jiakun. Prony Method Implementation Based on MATLAB. - Chinese Science and Technology Information Technology, 2007, No 4, 128-129.

12. Yifeng, Ding, Ha o zhong Cheng, Ganyun Lv, Yong Zhan, Yibin Sun, Rong L u. Spectrum Estimation of Harmonics and Interharmonics Based on Prony Algorithm., Vol. 20, 2005, No 10, 94-97.

13. Danping, Jia, Zhuo Yuan, Hongli San. The Study of Fluorescence Thermal Measurement Based on DSP. - Procedia Engineering, Vol. 29, 2012, 2864-2868.

14. Dan ping, Jia, Z huo Yuan. The Application and Dynamic Temperature Test Methods of Micro Thin Film Resistor Sensor. -Advanced Materials Research, Vol. 651, 2013, 511-516.

15. Zhang, Y., K. T. V. Grattan, A. W. Palmer. Spectral Characteristics and Effects of Heat Treatment on Intrinsic Nd-Doped Fiber Thermometer Probes. - Review of Scientific Instruments, 1997, No 68, 3442-3445.

16. Xia oling, Xia, Liw en Z hu. Fiber Optic Fluorescence Temperature Sensor. -Xiaogan College Journal, 2005, No 25, 78-81. 